\title{
Dynamic Tuning Retransmission Limit of IEEE 802.11 MAC Protocol for Networked Control Systems
}

\author{
Jia Bai, Emeka P. Eyisi, Yuan Xue, Xenofon D. Koutsoukos \\ Department of Electrical Engineering and Computer Science \\ Vanderbilt University \\ Nashville, TN, USA \\ \{jia.bai, emeka.p.eyisi, yuan.xue,xenofon.koutsoukos\}@vanderbilt.edu
}

\begin{abstract}
Building networked control systems over wireless networks is an extremely challenging task, as the wireless communication characteristics such as random packet losses and delay, significantly affect the stability and the performance of the control systems. We present a novel approach to the design of wireless networked control system. This approach decomposes the design concerns into two factors and addresses them separately in two design spaces - stability of the system is ensured using a passivity-based architecture at the control layer, while the performance of the system is optimized at the communication layer by adjusting the network operation parameters. This paper focuses on the design of IEEE 802.11-based wireless network. In particular, we present a MAC controller that dynamically adjusts the retransmission limit to track the optimal trade-off between packet losses and transmission delays and thus optimizes the overall control system performance. Simulation results show that our approach significantly improves the performance of the networked control systems.
\end{abstract}

\section{INTRODUCTION}

The integration of physical systems through computing and networking has become a trend now known as CyberPhysical Systems (CPS). Many CPS such as automotive vehicles and distributed robotics, are monitored and controlled by Networked Control Systems (NCS) which exchange information among sensors, controllers and actuators over a communication network. Wireless network is gaining increasing popularity with NCS, as it provides great convenience in terms of deployment and mobility support. Yet building NCS over wireless networks is an extremely challenging task. The wireless communication characteristics, such as random packet loss, time-varying delay and limited channel capacity, significantly affect the stability and the performance of the control systems.

This research was sponsored by the U.S. Army Research Office and Lockheed Martin and was accomplished under Cooperative Agreement W911NF-10-1-0005. The views and conclusions contained in this document are those of the authors and should not be interpreted as representing the official policies, either expressed or implied, of the Army Research Laboratory or the U.S. Government. The U.S. Government is authorized to reproduce and distribute reprints for Government purposes notwithstanding any copyright notation hereon. This work is also supported in part by the National Science Foundation under Grant CNS-1035655 and CCF-0820088.
Two major approaches have been investigated in the existing literature to address the challenges in building wireless networked control systems. One approach, independently of the network protocol design, investigates the design of the control layer (e.g., controller). The goal is to achieve a desired control system performance despite of the underlying network difficulties. For example, several works [1], [2] have been done to ensure the stability of the NCS in presence of packet losses and time-varying delay. Other works have focused on improving the performance of the NCS [3], [4], [5], [6], [7]. Yet without any support from the network, it is quite hard for the control layer to achieve stability and optimal performance simultaneously. For the works that ensure the NCS stability [1], [2], the issue of performance degradation is not addressed. For the works that improve the NCS performance [3], [4], [5], [6], [7], it is not clear whether they can achieve stability in wireless environment. The other approach is to perform a co-design of the control layer and the communication layer (e.g., network protocols) [8], [9], [10], [11], [12]. While this approach can achieve both stability and optimal performance of NCS, its design inevitably involves too many interactions between the control and the communication layers, which prevents efficient layer abstraction and encapsulation and also hinders broader adoption.

To address the above open issues, we present a novel approach to the design of wireless networked control system. This approach decomposes the design concerns into two factors and addresses them separately in two design spaces - stability of the system is ensured through controller design at the control layer; performance of the system is optimized through adjusting network protocol parameters at the communication layer. At the control layer, we leverage our previous work on using a passivity-based architecture in designing NCS that is robust to network delay and packet loss [2], [13]. In this paper, we focus on studying the impact of MAC layer packet retransmission on the performance of the passive controller and investigating the optimal design of retransmission strategies.

In IEEE 802.11 MAC protocol, a frame will get retransmitted up to a certain limit, if it is lost due to random channel 
errors. It is obvious that allowing a higher retransmission limit increases the chance of successful packet transmissions at the cost of longer packet transmission delays; while a lower limit will result in a larger packet loss probability with smaller delays for delivered packets. Since both packet loss and transmission delay have negative impact on the performance of the controller, the key questions we would like to answer are, what is the packet retransmission limit that optimizes the controller performance and how to achieve it. We consider a passive controller which produces a trajectory for the plant (a robotic arm in our system) to track and define the performance of this NCS as its absolute tracking error. We observe that the relationship between the NCS performance and the MAC retransmission limit can be characterized by convex functions depending on the channel error probability. Using this convex property, we design a heuristic control algorithm that dynamically adjusts the MAC retransmission limit to track the optimal retransmission limit under time-varying channel errors. Simulation results show that the MAC controller can converge quickly to a proper retransmission limit which optimizes the performance of the control system.

The main contributions of this paper are as follows. First, we present a novel approach to NCS design. By ensuring the stability of NCS at the controller layer and optimizing its performance at the communication layer, this approach is able to achieve both design goals of NCS while maintaining a clean cross-layer interaction. Second, we present a control algorithm that dynamically adjusts the MAC retransmission limit to track the best trade-off between packet loss and delay that optimizes NCS performance.

The rest of the paper is organized as follows. The system models are described in Section II. Section III shows the observation of the effect of loss and delay to the performance of the NCS. The MAC controller is designed in Section IV. The experiment evaluation results are presented in Section V. Finally, Section VI summarizes this paper.

\section{SYSTEM MODELS}

We consider a networked control system consisting of a controller and a plant communicating through a UDP connection over an IEEE 802.11-based wireless network. The controller controls the plant, which is a robotic arm, to follow.

\section{A. Control Layer}

Fig. 1 shows the structure of the system in the control layer. The figure depicts a passive control architecture for the digital control of a continuous plant, over a wireless Local Area Network (LAN). In [2], the architecture is shown to be passive by design, which means it ensures stability of the NCS in the presence of network uncertainties such as time varying delays and packet losses. A control system is considered stable if its output will stay bounded for any

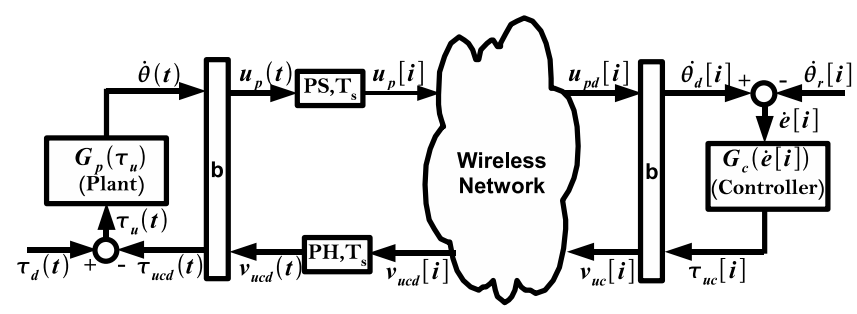

Figure 1: Passivity Based Control Architecture Over Wireless Networks

bounded input, and the system performance is considered as how fast and accurate the plant can track the control signal within the bound. Using this passive architecture allows us to focus solely on system performance. We provide a brief description of this architecture, and refer the reader to [2] for a detailed description and proofs pertaining to the passive control architecture.

In Fig. $1, G_{p}\left(\tau_{u}\right)$ is the plant system to be controlled. The plant is a continuous linear time-invariant system and the composite dynamics of the plant is by design, strictly output passive. The plant system takes the torque control command $\tau_{u}(t)$ as input, and outputs velocity $\dot{\Theta}(t) . G_{c}(\dot{e}[i])$ denotes the digital controller which controls the plant to behave in a desired manner. The digital controller is a discretetime linear time-invariant system and is also designed to be strictly output passive. The controller takes as input, the error velocity $\dot{e}[i]$ between the reference and the plant output, and outputs torque command $\tau_{u c}[i]$.

The block $b$ transforms the power variables (i.e., the direct input and output of plant and controller) into wave variables for communication over a wireless network. These wave variables preserve the passivity of the system. On the plant side, the wave variable $v_{u c d}(t)$ and the velocity measurement $\dot{\theta}(t)$ are considered inputs to the wave transform block and the wave variable $u_{p}(t)$ and delayed torque command $\tau_{u c d}(t)$ are considered outputs of the wave transform block. On the controller side, the wave variable $u_{p d}[i]$ and the control torque $\tau_{u c}[i]$ are considered inputs to the wave transform block and the wave variable $v_{u c}[i]$ and delayed velocity measurement, $\dot{\theta}_{d}[i]$ are considered outputs of the wave transform block.

The $\left[P S, T_{s}\right]$ and $\left[P H, T_{s}\right]$ blocks represent the passive sampler and passive hold respectively. The passive sampler, at a sampling time $T_{s}$, interconnects the plant to the digital controller. It converts the continuous wave variable $u_{p}(t)$ to an appropriately scaled discrete wave variable $u_{p}[i]$. The passive hold, on the other hand, converts the discrete time wave variable $v_{u c d}[i]$ to an appropriately scaled wave variable $v_{u c d}(t)$ which is held for $T_{s}$ seconds.

\section{B. Communication Layer}

The controller and the plant are implemented on two separated nodes which send their commands and measurements (precisely wave variables) using UDP protocol. The UDP 
packet rate naturally corresponds to the sampling rate of the controller.

The two nodes communicate with each other directly over wireless channel using the IEEE 802.11 MAC protocol. Here we consider a wireless channel with random errors. In IEEE 802.11, if a frame is corrupted due to channel errors, it will be retransmitted. When the number of retransmission reaches a certain limit, the frame will be dropped. According to [14], the value of retransmission limit depends on the size of the frame. For frames with sizes larger than RTSThreshold, LongRetryLimit of 4 times will be used; for frames smaller than RTSThreshold, ShortRetryLimit of 7 times will be used. To simplify the system model, we disable the RTS/CTS mechanism by setting RTSThreshold to a very large value in the IEEE 802.11 MAC protocol.

It is obvious that the MAC retransmission strategy may affect the NCS performance. Given a packet loss probability, allowing a larger retransmission limit increases the chance of successful transmission of a particular packet. However, it can also result in a longer delay in the packet transmission, which can be harmful especially if the system is delaysensitive. On the other hand, if a small retransmission limit is used, the packets may experience a higher drop rate, which can also degrade the system performance especially if the system is loss-sensitive. Yet, to identify the optimal MAC retransmission strategy, we need to investigate how much the delay and the loss will be factored into the NCS performance.

\section{OBSERVATIONS}

To understand how the network loss and delay may affect the performance of the NCS and how retransmission strategy should be designed to minimize such effect, we perform a set of experiments using ns-2 simulator.

\section{A. Methodology}

We implement the passive control architecture on top of IEEE 802.11 wireless network in ns-2 simulator. In our experiment, the sampling rates of the plant and the controller are both 20 samples/sec, which is also the UDP packet rate. The packet size is 210 bytes. The wireless network has a capacity of $1 \mathrm{Mbps}$. Each simulation runs for 100 seconds.

The velocity of the plant system $G_{p}\left(\tau_{u}\right)$ tracks a sinusoidal reference input $\dot{\theta}_{r}[i]=\sin (\omega i)$ with $\omega=\frac{2 \pi}{10}$. The performance of the system is evaluated using the instantaneous tracking error $J[i]=\left|\dot{\theta}[i]-\dot{\theta}_{r}[i]\right|$, where $\dot{\theta}[i]$ is the plant's output and $\dot{\theta}_{r}[i]$ is the reference input the plant is supposed to track. $J[i]$ demonstrates the tracking ability of the system.

In what follows, we first inspect how the network loss affects the plant output when network delay is negligible, then test the effect of network delay to the NCS in a loss free condition. We finally investigate the effect of the MAC retransmission limit on the NCS, which will establish the basis of our control algorithm for retransmission limit.

\section{B. Effect of Packet Loss}

In this experiment, we disable the retransmission mechanism of IEEE 802.11 MAC so that each packet will only be transmitted once. In this case, the packet error directly translates to a packet loss.

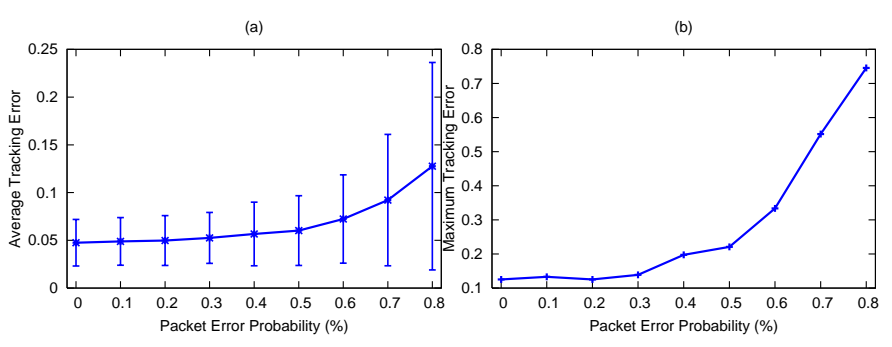

Figure 2: Impact Of Loss Rate On System Performance

Fig. 2 demonstrates the system performance with different packet error probabilities. Fig. 2(a) shows the average tracking error $\bar{J}$ over all sampling points with standard deviation $J_{d}$. Fig. 2(b) shows the maximum tracking error $J_{m}$ experienced out of all samples corresponding to different error probabilities. With the increase of the error probabilities, $\bar{J}, J_{d}$ and $J_{m}$ all increase. When the error probability is small, only a few packets are dropped. If the samples are exchanged frequently enough, the plant and the controller can still keep track of each other's status. However, when too many packets are dropped, the plant cannot interpret the control command correctly, while the controller no longer has the right velocity information of the plant. For example, when the packet error probability is $70 \%$, the errors suddenly become very large. Further experiments using different passive controllers on different signals and with different sampling rates all show the same trend. Yet the exact mathematical relation between error probability and tracking error varies depending on these system parameters (e.g., signal, sampling rate).

\section{Effect of Network Delay}

In this experiment, the controller and the plant work in a loss free network. A varying amount of delay $D$ is introduced before the packet transmission at the MAC layer. The value of $D$ can be regarded as the time a packet spends in channel contention. Thus it is an indicator of the intensity of background traffic in the wireless network. Fig. 3 shows the NCS performance in terms of average and maximum tracking error under different values of $D$.

We observe that when $D$ is small, the system performance does not change much. When exceeding certain value, the performance degrades significantly. Consider that the controller is a discrete time system, when the delay is smaller than one discrete time step, the controller can still receive the signals for the next sampling period in time, so the performance does not deteriorate. However, when a larger delay is experienced, signals cannot reach the other 

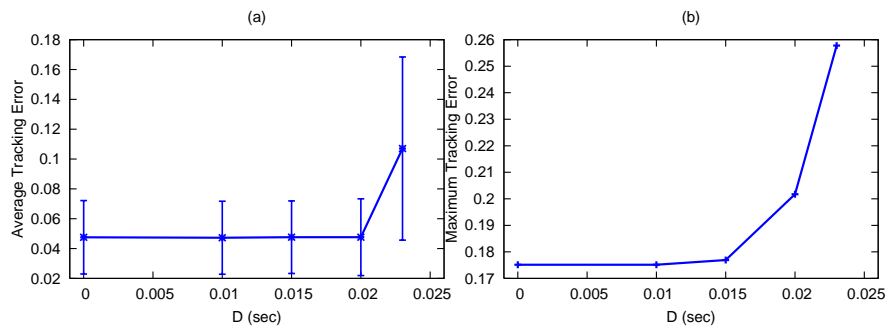

Figure 3: Impact Of Transmission Delay On System Performance

end within one sampling period. In this case, When the controller receives a signal from the plant, the state of the continuous plant may have already changed considerably. But the controller will still produce a control signal for the plant using the received plant state information. This control signal will also experience transmission delay before it arrives at the plant. When these two delays are combined, the plant will deviate more from the expected trajectory. It is important to note that increasing $D$ also reduces the departure rate of the packets and may cause queueing delay (e.g., when $D=0.023 s^{1}$ ), so the actual delay experienced by the system is much larger. Due to this reason, this delay threshold (0.02 in this experiment) highly depends on the sampling rate, the signal of the system and the channel capacity. Further experiments on different signals and with different sampling rates validate the same observation.

\section{Effect of MAC retransmission}

In IEEE 802.11-based wireless networks, packet loss will be recovered through retransmission up to a limit. As a result, a packet may experience higher delay before getting successfully transmitted. In the first two experiments, we have demonstrated that increasing either the network loss or delay will harm the performance of the control system. To achieve the optimal NCS performance, the retransmission strategy needs to be carefully designed to provide the best trade-off between the packet delay and the loss. Here, we exam the impact of MAC retransmission strategy by varying the retransmission limit and measure the system performance.

Fig. 4 shows the average and maximum tracking errors of the system under different retransmission limits, with varied packet error probabilities and delay parameters $D$. We observe that the relationship between the tracking error and the retransmission limit follows a convex curve in all experiments. When the retransmission limit is small, high packet loss rate leads to relatively large tracking errors. When the limit is too high (e.g., 7 defined as ShortRetryLimit in IEEE 802.11), the tracking error raises due to large delay. Experiments with different signals and sampling

\footnotetext{
${ }^{1}$ To have a controlled environment, where only the impact of delay is assessed, the queue length and the value of $D$ are carefully chosen in this experiment, making sure no queuing loss is incurred.
}
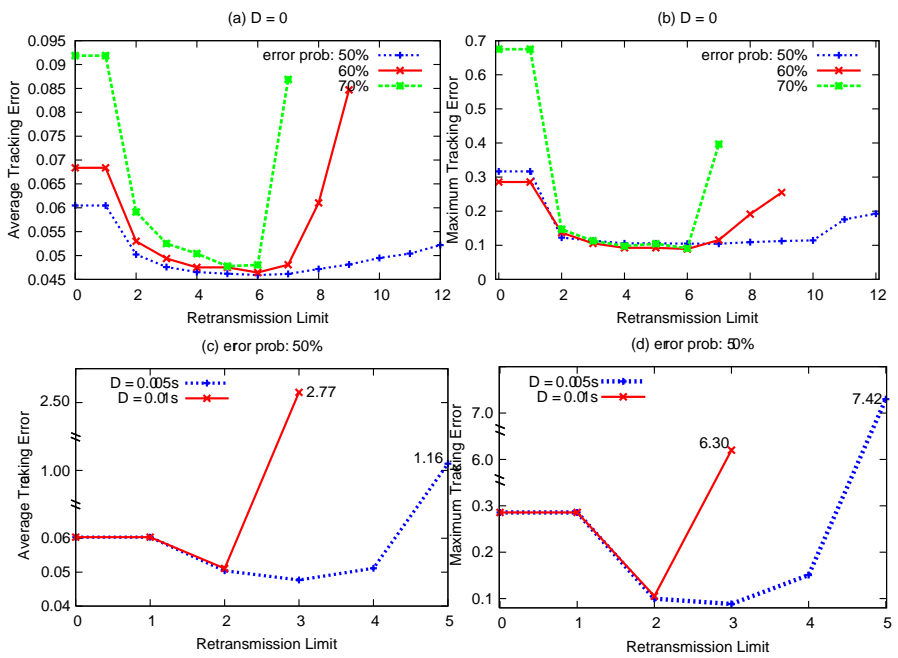

Figure 4: Impact Of Retransmission Limit On System Performance

rates confirm the convex relation between the retransmission limit and the tracking error. This observation implies that there exists a unique optimal value for retransmission limit. Yet this optimal value varies depending on the channel error probability, background traffic, signal property, etc. We summarize our observations below:

- The NCS performance based on passive controller is negatively affected by network factors, including packet losses and transmission delays.

- The MAC-layer packet retransmission limit and the NCS performance follows a convex relation, which shows the existence of a unique value for retransmission limit that optimizes the NCS performance. This optimal value depends on many control system properties, such as sampling rate, signal type, as well as network factors, such as channel error probability, background traffic, etc.

- The fixed retransmission limit (4 as LongRetryLimit, 7 as ShortRetryLimit) used in IEEE 802.11 is not optimal for the NCS performance, considering the dynamics in wireless network with bursty traffic and fluctuating channel conditions. To achieve the optimal NCS performance, the retransmission limit needs to be dynamically adjusted based on the system property.

\section{MAC CONTROL DESIGN}

In this section, we present a MAC-layer controller that dynamically adjusts the retransmission limit under different network conditions. Most NCS systems define a maximum performance error they can tolerate. We use $\tilde{J}$ to represent this threshold. Our MAC controller can achieve the following two goals: (1) keep the NCS performance within this error threshold; (2) minimized the NCS performance error (when the error threshold is not achievable, or set to a very small value). 


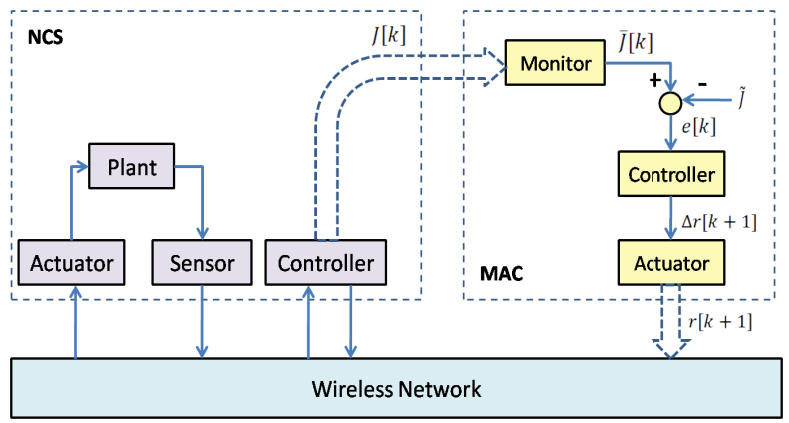

Figure 5: MAC Controller Architecture

Fig. 5 is an overview of the MAC controller architecture, which consists of a monitor, a controller and an actuator. The monitor resides on the same node as the NCS controller. It interfaces with the NCS and measures the average track error $\bar{J}[k]$ of the last $m$ samples in the current MAC sampling period $k^{2}$. The monitor will then derive the difference $e[k]$ between $\bar{J}[k]$ and $\tilde{J}$ as $e[k]=\bar{J}[k]-\tilde{J}$ and pass it to the controller. Let $r[k+1]$ be the retransmission limit that will be used in the MAC layer at time $k+1$ and $\Delta r[k+1]$ be the adjustment of $r[k+1]$. The controller will compute $\Delta r[k+1]$ based on $e[k]$, and send it to the actuator. The actuator interfaces with the wireless network and tunes the retransmission limit to $r[k+1]=r[k]+\Delta r[k+1]$. For the node on which the NCS controller resides, the actuator will directly pass the new retransmission limit to the MAC layer. For the node on which the plant resides, the MAC actuator sends the new retransmission limit information on a separate packet or piggyback on a data packet.

The MAC controller at the communication layer and the "main" NCS controller at the control layer form a timescale-decomposed system, where the MAC controller is the slow system that evolves with a larger time scale and operates with a lower sampling rate. This allows the NCS performance to converge with the new retransmission limit.

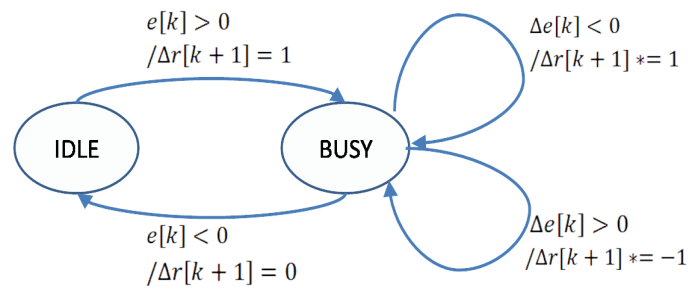

Figure 6: MAC Controller Design

To indicate whether the NCS system is within its error threshold, the MAC controller maintains two states, IDLE and BUSY, as in Fig. 6. Initially the controller is at the IDLE state. If the measured error is within the threshold (i.e., $e[k]<0$ ), the controller will remain at the IDLE state with

\footnotetext{
${ }^{2}$ As explained below, the sampling period of the MAC controller is larger than the sampling period of the NCS controller.
}

$\Delta r[k+1]$ set to 0 , meaning no changes to the retransmission limit. When the measured error is greater than the threshold (i.e., $e[k]>0$ ), the controller will transit from the IDLE state to the BUSY state and set $\Delta r[k+1]$ to 1 .

At the BUSY state, the MAC controller will determine the change of retransmission limit for time slot $k+1$ based on the change of the tracking error from time $k-1$ to $k$ $\Delta e[k]=\tilde{e}[k]-\tilde{e}[k-1]$. If the tracking error becomes smaller (i.e., $\Delta e[k]<0$ ), which means the previous change of the retransmission limit $\Delta r[k]$ decreases the tracking error of the NCS, the controller will keep the same change to the limit:

$$
\Delta r[k+1]=\Delta r[k]
$$

If $\Delta e[k]>0$, which means $\Delta r[k]$ increases the tracking error, the controller will then change the retransmission limit towards the opposite direction:

$$
\Delta r[k+1]=-\Delta r[k]
$$

Whenever the tracking error falls below $\tilde{J}$, the controller will transit the state back to IDLE.

Discussion. We make the following important notes about the MAC controller design:

- This MAC controller will adjust the retransmission limit so that the NCS performance error is within a predefined threshold. When there are multiple values of the retransmission limit that can enable the system to perform within the error threshold, the MAC controller may bring the limit to any of these values.

- If the threshold is too small that no feasible retransimission limit value can bring the NCS system within this threshold, the MAC controller will bring the retransmission limit close to the optimal value where the NCS performance error is minimized. This is ensured by the convexity property in the relationship between retransmission limit and the NCS performance as we have demonstrated in Section III. We can exploit this feature to achieve the goal of optimal NCS performance by manually setting the threshold to a very small value.

- In its current design, though the MAC controller can bring the retransmission limit to the optimal value, it can not stay at this point. Rather it will oscillate around it. To ensure the controller stabilizes at the optimal value, we improve its design in two ways. First, a counter is used to count the number of times the controller oscillates around one retransmission limit. If the counter exceeds a certain value, we consider the limit as the optimal value and fix the retransmission limit to it. After some time, the MAC controller will resume to the BUSY state in case the optimal retransmission limit has changed. 


\section{Simulation Study}

\section{A. Simulation Setup}

We implement the MAC controller in the ns- 2 simulator and use it to evaluate the NCS performance under a variety of network scenarios. The NCS used in the experiment consists of a passive controller and a plant. It has the same configuration as the one presented in Section III. The error threshold $\tilde{J}$ is set to 0.1 in all experiments. Two aspects of the NCS system will be examined: (1) performance of the MAC controller in terms of the convergence behavior of the retransmission limit, and (2) performance of the overall NCS in terms of the absolute tracking error of the plant output. We will compare the performance of NCS that operates with our MAC controller which dynamically adjusts the retransmission limit, with the NCS that operates over traditional IEEE 802.11-style MAC, where retransmission limit is fixed.

\section{B. Simulation Results}

1) Impact of Initial Value of Retransmission Limit: In this experiment, the controller and the plant operate and communicate in a network with no background traffic. The wireless channel error probability is $83 \%$.
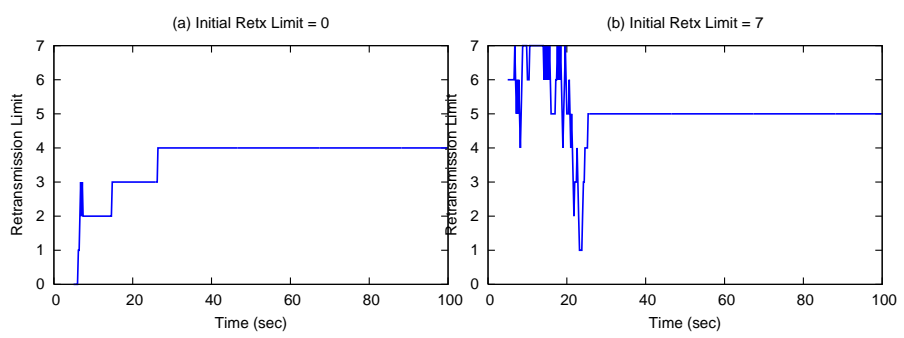

Figure 7: Convergent Retransmission Limit With Different Initial Values

Fig. 7(a) and (b) display the retransmission limit adjusted by the MAC controller from initial values of 0 and 7 respectively. In both cases, the MAC controller converges after 25 seconds. The retransmission limit has different stable values. This is because the tracking error of the NCS performance is below the error threshold for both retransmission limits of 4 and 5 . When the initial value is 7, a larger delay may cause a long queue in the MAC, and the change of the retransmission limit cannot be reflected onto the NCS performance immediately. For the rest of the simulation, the initial values of the retransmission limit are all 0 .

2) Impact of Background Traffic: Background traffic in the wireless network will increase the time a packet spends on contending for the medium access, which in turn will increase the packet delay. We now study how the background traffic will affect the behavior of the MAC controller and the overall system performance. With 50\% packet error probability, three pairs of background traffic nodes are introduced to the network. The traffic between each pair of nodes follows a poisson distribution with rate of 200 packets/sec and packet size of 210 bytes.
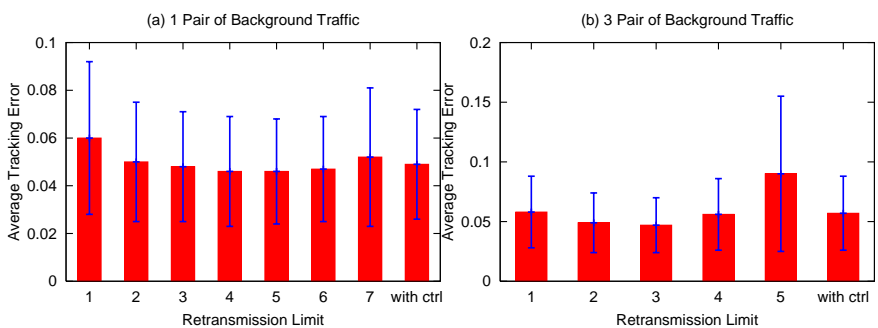

Figure 8: Tracking Errors with Different Background Traffic

Fig. 8 (a) and (b) show the tracking errors of using the MAC controller and using different fixed retransmission limits. Note that In Fig. 8(b), the retransmission limits of 6 and 7 are not shown as the tracking errors are in much larger scales than the others. We could see that the optimal values of retransmission limit differ under different background traffic. The MAC controller converges to the optimal retransmission limits of 4 and 3 , respectively in both scenarios. We could also see that the NCS performance under the MAC controller is close to the optimal fixed retransmission limit. The slight difference is due to the suboptimal NCS performance during the initial transient phase when MAC controller is adjusting the retransmission limit.

3) Impact of Channel Error Probability: This experiment tests the impact of packet error probability on the MAC controller and the NCS performance. With one pair of poisson background traffic as in the previous experiment, we simulate the scenarios with the packet error probability of $40 \%$ and $80 \%$.
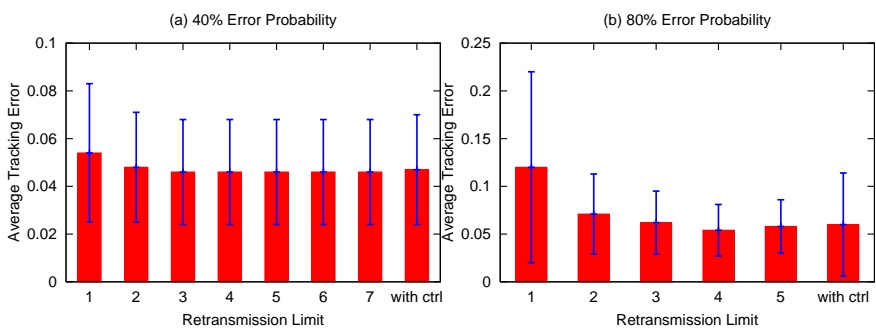

Figure 9: Tracking Errors With Different Error Probability

When the packet error probability is $40 \%$ as in Fig. 9, several retransmission limits can provide optimal performance for the MAC controller. In this case, the MAC controller can converge to any of the retransmission limits to achieve the optimality of the NCS. Here it converges to 3. When the error probability increases to $80 \%$, the MAC controller must adjust to the correct retransmission limit to best support the NCS, and here it converges to 5. The deviation of the tracking error in the MAC controller from the optimal fixed 
retransmission limit is due to the initial adaptation of the MAC controller.

\section{CONCLUSIONS}

This paper studies the design of wireless networked control system by decomposing the concerns into two design spaces. The NCS is first guaranteed stable by using a passive control structure in the control layer. The overall NCS performance is then optimized by adjusting the network protocol parameters. Through experiment study, this paper reveals the important convex relationship between the retransmission limit of IEEE 802.11 and the NCS performance. Based on this observation, we present a MAC-layer control algorithm that dynamically tunes the retransmission limit so that the NCS performance can be either bounded by an error threshold or optimized. The results of the simulation study show that our MAC controller can achieve robust adaption and enable desirable NCS performance under a variety of network conditions including highly lossy channel and with heavy background traffic.

\section{REFERENCES}

[1] M.S. Branicky, S.M. Phillips, and W. Zhang, "Stability of networked control systems: Explicit analysis of delay," in Proc. of the American Control Conference, 2000, pp. 23522357.

[2] N. Kottenstette, X. Koutsoukos, J. Hall, J. Sztipanovits, and P. Antsaklis, "Passivity-based design of wireless networked control systems for robustness to time-varying delays," in Proc. of RTSS, 2008, pp. 15-24.

[3] Y. Tipsuwan and M. Chow, "Network-based controller adaptation based on qos negotiation and deterioration," Proc. of IECon 01, pp. $1794-1799,2001$.

[4] P. Marti, J. Yepez, M. Velasco, R. Villa, and J. M. Fuertes, "Managing quality-of-control in network-based control systems by controller and message scheduling co-design," IEEE/ACM Trans. On Industrial Electronics, vol. 51, no. 6, pp. 1159-1167, 2004.

[5] Y. Tipsuwan and M. Chow, "Control methodologies in networked control systems," Control Engineering Practice, vol. 11, no. 10, pp. 1099-1111, 2003.

[6] A. Ray and Y. Halevi, "Integrated communication and control systems: Part ii-design considerations," 1988, vol. 110, pp. 374-381.

[7] J. Nilsson, "Real-time control systems with delays," Ph.D. Dissertation, Lund Institute of Technology, 1988.

[8] X. Liu and A. Goldsmith, "Wireless medium access control in networked control systems," 2004.

[9] S. Nethi, M. Pohjola, L. Eriksson, and R. Jäntti, "Simulation case studies of wireless networked control systems," in PM2HW2N, 2007, pp. 100-104.
[10] J. Colandairaj, G. Irwin, and W. Scanlon, "Analysis of an ieee 802.11 b wireless networked control system," in Proc. of the 1st NeCST Workshop on Networked Control Systems and Fault Tolerant Control, 2005, pp. 19-25.

[11] L. Xiao, M. Johansson, H. Hindi, S. Boyd, and A. Goldsmith, Joint optimization of wireless communication and networked control systems, vol. 3355, chapter Switching and Learning in Feedback Systems, pp. 248-272, Springer Berlin/Heidelberg, 2005.

[12] G. Nikolakopoulos, A. Panousopoulou, and A. Tzes, "Experimental controller tuning and qos optimization of a wireless transmission scheme for real-time remote control applications," Control Engineering Practice, vol. 16, no. 3, pp. 333 - 346, 2008.

[13] N. Kottenstette and P. J. Antsaklis, "Stable digital control networks for continuous passive plants subject to delays and data dropouts," in Proc. of the 46th IEEE Conference on Decision and Control, 2007, pp. 4433 - 4440.

[14] "Part 11: Wireless lan medium access control (mac) and physical layer," 2007. 